\title{
A case report of Morvan syndrome, the unique clinical pattern of a rare disease
}

\author{
Diana Anamaria Epure ${ }^{1}$, Mihaela Roxana Ioghen', Eugenia Roza ${ }^{1,2}$, \\ Raluca Ioana Teleanu ${ }^{1,2}$ \\ 1"Dr Victor Gomoiu" Children's Hospital, Bucharest, Romania \\ 2"Carol Davila" University of Medicine and Pharmacy, Bucharest, Romania
}

\begin{abstract}
Morvan syndrome is a rare autoimmune disorder mediated by antibodies against voltage-gated potassium channels (VGKC) and characterized by the involvement of both the peripheral and central nervous system. We report a case of Morvan syndrome. The patient presented with peripheral nerve hyperexcitability, dysautonomia and neuropsychiatric symptoms. The diagnosis was based on the unique clinical presentation that was highly suggestive of Morvan syndrome and confirmed by positive VGKC antibodies. In this paper we try to highlight the importance of clinical skills in the diagnosis and decision-making concerning the treatment process.
\end{abstract}

Keywords: Morvan syndrome, peripheral nerve hyperexcitability, dysautonomia, encephalopathy, VGKC

\section{INTRODUCTION}

Morvan syndrome, also called Morvan's lary chorea, is a rare autoimmune and life-threatening neurologic disorder mainly characterized by peripheral nerve hyperexcitabilty, dysautonomia and encephalopathy with severe insomnia $(1,2)$.

It may involve the peripheral nervous system (e.g. hyperexcitation - painful cramps, myokymia, fasciculations, neuropathic pain, the autonomic nervous system (e.g. hyperhidrosis, variations in blood pressure, cardiac arrhythmias, tachycardia, urinary complaints, constipation), the central nervous system (e.g. encephalopathy - hallucinations, confusion, delusions, agitation, amnesia, severe insomnia, myoclonus). It may also present with systemic features such as weight loss, skin lesions or pruritus (1,3-5).

The disorder is regarded as autoimmune in nature and it is associated with autoantibodies against voltage-gated potassium channels (VGKC) (2). VGKC are transmembrane channels complexed with various proteins, such as leucine-rich glioma inactivated protein 1 (LGI1), contactin-associated protein 2 (CASPR2) and contactin-2. The autoantibodies are directed against one or two of these proteins, mainly CASPR2. The association with a certain type of protein leads to a clinical presentation. VGKC repolarize active neurons to the resting state after each nerve impulse, thus playing an essential role in modulating excitability in the central and peripheral nervous system $(5,6)$.

It was demonstrated that LGI1 and CASPR 2 are expressed throughout the central nervous system in the thalamic neurons, hypothalamic orexin neurons (orexin regulates feeding behaviour and sleep/ wakefulness cycle), locus coeruleus neurons (promotes wakefulness, attention and arousal) and the raphe nuclei (major source of serotonin, which is involved in various neuropsychiatric disorders) $(5,7,8)$. LGI1 is also located particularly in the hippocampus and CASPR2 in the hippocampus and cerebellum (9). The physiological role of the VGKC and the localizations of the proteins that are targeted in the Morvan syndrome could partly explain the clinical presentation discussed above. 
In many cases Morvan syndrome is paraneoplastic, being especially associated with thymoma and small cell lung cancer but it can also occur in the absence of any cancer. It can also be associated with myasthenia gravis (10).

Treatment options include plasma exchange, corticosteroids, intravenous immunoglobulins, immunosuppression with azathioprine / cyclosporine/ cyclophosphamide $(3,10)$.

We present one clinical case that we encountered in our practice.

\section{CASE REPORT}

A 13-year old boy presented with thigh and calf fasciculations, inferior limb muscle pain, insomnia (2-3 hours of sleep/night), marked anxiety, agitation, hyperhydrosis, weight loss (15 kg in 2 months), diarrhea (up to 20 stools/day), frequent micturitions and tachycardia (120 bpm).

Three months prior to being admitted into our clinic, he developed muscle pain in the inferior limbs following an episode of hives and moderate workout. Several days later, the pain grew in intensity and associated profuse sweating, tremor, chills, nausea and inferior limb fasciculations.

Hyperthyroidism was considered as a diagnosis as the patient presented with anxiety, agitation, hyperhidrosis, weight loss and tachycardia. It was excluded, as thyroid hormones, thyroid ultrasound and thyroid peroxidase antibodies were found to be within normal range.

The combination of insomnia, agitation, anxiety, hyperhidrosis and tachycardia pointed towards anxiety disorder as a differential diagnosis. The patient underwent psychiatric evaluation and it was concluded that the disease was not psychiatric in nature.

A pediatric neurology consultation revealed some important clinical features (a) muscular signs and symptoms: fasciculations, muscular pain, myokymias, (b) neuropsychiatric symptoms: severe insomnia, asthenia, sadness and (c) autonomic signs and symptoms: hyperhidrosis, blood pressure instability, tachycardia episodes, and constipation. This combination of signs and symptoms lead to the clinical diagnosis of Morvan syndrome.

The electromyographic studies revealed myokymias and intense positive doublet and triplet patterns in all muscle groups. This aspect was suggestive for peripheral nerve hyperexcitability, a key feature of Morvan syndrome. Anti VGKC antibodies testing showed a value of $364 \mathrm{pmol} / 1$ (normal range $<85 \mathrm{pmol} / \mathrm{l}$ ), which reinforced the diagnosis of Morvan syndrome. As Morvan syndrome might be paraneoplastic in nature, a screening for neoplastic disease was performed (cerebral MRI, mediastinum MRI, whole body MRI) and it was unremarkable. The combination of peripheral nerve hyperexcitability symptoms plus EMG and positive anti-VGKC antibodies are also the key features of Isaacs' syndrome, but Morvan also includes central nervous system symptoms and dysautonomia, which were present in our patient.

He was started on carbamazepine in progressively increasing doses and $6 \mathrm{mg}$ melatonin which resulted in some improvement of insomnia, remission of the profuse sweating, normalization of gastrointestinal transit and slight improvement of muscle pain.

Treatment with prednisone $0,54 \mathrm{mg} / \mathrm{kg} /$ day, ranitidine $300 \mathrm{mg} /$ day, carbamazepine $600 \mathrm{mg} /$ day, melatonin $10 \mathrm{mg} /$ day was unsuccessful. Subsequently, the patient was admitted to our clinic and was started on intravenous corticosteroids (pulse IV Metilprednisolone therapy, $1 \mathrm{~g}$ /day for 3 days), melatonin $5 \mathrm{mg}$ /day and the dose of carbamazepine was increased to $900 \mathrm{mg} /$ day with notable improvement: insomnia remission and amelioration of fasciculations and muscle pain.

\section{DISCUSSION}

Morvan syndrome was named after the physician Auguste Morvan, who described the disease

To date, few cases of Morvan syndrome have been described and reported in the literature worldwide. In the case of our patient, the diagnosis was delayed by 2 months. For a long time neurologists were seen as diagnosticians who had only very few treatment options for their patients. Due to the development of new drugs and the elucidation of neurological disorders in more depth, this view is no longer up to date, perhaps in the of neuroimmunology more than in any other neurological

Although it is a rare disorder, early recognition of Morvan syndrome is important because it is 
responsive to immunomodulatory therapy. The diagnosis is established mainly based on the unique clinical combination of peripheral nerve hyperexcitability, dysautonomia and encephalopathy with severe insomnia. Every time this constellation of symptoms is seen in a patient, antibodies against VGKC should be tested.

Our patient presented with psychiatric symptoms such as anxiety, agitation and insomnia. Considering the fact that the clinical picture of Morvan syndrome often includes psychiatric symptoms, it might be misdiagnosed as a pure psychiatric disorder

If the clinical diagnosis is very suggestive, appropriate treatment can be started while awaiting results for the antibody titer because the diagnosis is mainly clinical and seronegative Morvan syndrome cases were also described in the literature $(6,11,12)$.

Considering the fact that Morvan syndrome can be paraneoplastic in nature, screening for neoplastic disease should also be performed. Thymoma is the type of tumor to be taken into consideration because it has been described as the most frequently associated tumor (10). Our patient did not have paraneoplastic Morvan syndrome as we excluded the presence of any tumor.

A multidisciplinary collaborative approach is recommended, especially involving a cardiologist, because autonomic symptoms can lead to blood pressure instability and arrhythmia (5). Our patient presented with tachycardia and blood pressure instability, autonomic symptoms of the Morvan syndrome.

Concerning the treatment, oral corticosteroids alone were enough to relieve symptoms in some cases, but most of the time intravenous pulse corticosteroids are needed. Our case is a good example, as oral corticosteroids were and the administration of intravenous pulse corticosteroids relieved the symptoms. The evolution of the disease without treatment is highly variable. Spontaneous remission was reported in some cases, but the natural history can also lead to exitus, which makes early diagnosis and treatment of the utmost importance.

\section{CONCLUSIONS}

Despite the fact that the diagnosis was supported by positive VGKC antibodies, the clinical ings (the unique combination of peripheral nerve hyperexcitability, dysautonomia and encephalopathy) were essential for the diagnosis as they guided the laboratory workout. Although history-taking and physical examination skills are often underestimated in the era of modern technology, our case represents an illustrative example that they represent the foundation of medical decision-making.

Conflict of interest: none declared Financial support: none declared

8. Samuels ER, Szabadi E. Functional neuroanatomy of the noradrenergic locus coeruleus: its roles in the regulation of arousal and autonomic function part I: principles of functional organisation. Curr Neuropharmacol. Bentham Science Publishers; 2008; 6(3):235-53.

9. Irani SR, Alexander S, Waters P, Kleopa KA, Pettingill $P$, Zuliani L et al. Antibodies to Kv1 potassium channel-complex proteins leucinerich, glioma inactivated 1 protein and contactin-associated protein-2 in limbic encephalitis, Morvan's syndrome and acquired neuromyotonia. Brain. 2010;133(9):2734-48.

10. Vale TC, Pedroso JL, Dutra LA, Azevedo L, Filho LHP, Prado LBF et al. Morvan syndrome as a paraneoplastic disorder of thymoma with anti-CASPR2 antibodies. Lancet. Elsevier Ltd; 2017; 389(10076):1367-8.

11. De Michele G, Filla A, Marano E, Rinaldi C, Russo CV. Course and outcome of a voltage-gated potassium channel antibody negative Morvan's syndrome. Neurol Sci. 2009;30(3):237-9.

12. Cottrell DA, Blackmore KJ, Fawcett PR, Birchall D, Vincent A, Barnard S, Walls TJ. Sub-acute presentation of Morvan's syndrome after thymectomy. J Neurol Neurosurg Psychiatry. 2004 Oct; 75(10):1504-5. 